\title{
Thermopower Peculiarities of Uniaxial-Strained Bismuth Nanowires
}

\author{
E. Condrea - A. Nicorici • \\ A. Gilewski · S. Matyjasik
}

Received: 25 July 2013 / Accepted: 26 December 2013 / Published online: 23 January 2014

C The Author(s) 2014. This article is published with open access at Springerlink.com

\begin{abstract}
We present the results of the investigations of the thermopower and electrical resistance of Pyrex-coated Bi nanowires. Here we show that there is a critical thickness of wire below which structural defects can strongly influence the charge transport in Bi nanowires, fabricated by the melt spinning method. At low temperature the contribution of holes to the thermopower dominates the electron contribution. Thermoelectric parameters of Bi nanowires may be controlled by applying a thermal treatment and/or a uniaxial pressure. A fairly high value of thermoelectric power factor was found under strain in the temperature range of 80-300 K, where the dominant mechanism contributing to the thermopower is diffusive thermoelectric generation with electrons as the majority carrier.
\end{abstract}

Keywords Wires · Uniaxial pressure - Thermopower · Power factor

\section{Introduction}

Currently nanowires are widely used as building elements in a variety of micro/nano devices. Interest in studying the transport properties of Bi nanowires (NWs) is supported by the theoretical prediction on enlarged thermoelectric efficiency of NWs, which results from the quantum size effect [1]. Although to date there has been no reliable experimental evidence showing an improved thermoelectric efficiency in $\mathrm{Bi}$

\footnotetext{
E. Condrea $(\varangle) \cdot$ A. Nicorici

Institute of Electronic Engineering and Nanotechnologies, Academy of Science of Moldova, 3/3 Academiei, 2028 Chişinău, Republic of Moldova

e-mail: condrea@nano.asm.md

E. Condrea · A. Gilewski · S. Matyjasik

International Laboratory of High Magnetic Fields and Low Temperatures,

Gajowicka 95, 51-421 Wroclaw, Poland
} 
Table 1 The thermopower discrepancy observed between experimental results for Bi wires obtained by different technological methods

\begin{tabular}{|c|c|c|c|}
\hline \multirow[t]{3}{*}{ Technological methods } & \multicolumn{2}{|c|}{ Thermopower (TEP) } & \multirow{3}{*}{ Temperatures $(T) \mathrm{K}$} \\
\hline & \multicolumn{2}{|c|}{ Carrier dominated transport ( $d$-diameter nm) } & \\
\hline & Positive (p-type) & Negative (n-type) & \\
\hline $\begin{array}{l}\text { Glass-coated Bi Nws [12] } \\
\text { Bi Nws [13] and } \\
\text { composites embedded } \\
\text { in porous anodic } \\
\text { alumina }\left(\mathrm{Bi} / \mathrm{Al}_{2} \mathrm{O}_{3}\right) \\
{[14]}\end{array}$ & $\begin{array}{l}d<800 \\
\text { Positive }\end{array}$ & $\begin{array}{l}d>800 \\
\text { Negative } \\
d=40 \text { and } 65\end{array}$ & $\begin{array}{l}77 \\
\text { Below } 30 \\
\text { Above } 30 \\
4.2-300\end{array}$ \\
\hline $\begin{array}{l}\text { Individial Bi Nws [2] } \\
\text { Bi whiskers [15] }\end{array}$ & Positive & Negative $(d=800-2000)$ & $\begin{array}{l}4.2 \\
4.2-300\end{array}$ \\
\hline
\end{tabular}

NWs, many published works indicate that the idea of studying pure Bi and Bi based crystals as a material with promising thermoelectric efficiency and high sensitivity to pressure is still attractive [2-7]. The prospect of the thermoelectric application of NWs will require the ability to control their parameters. In this context, Bi wires embedded in an individual glass capillary allow us to perform measurements of thermoelectric parameters in large ranges of temperature and elastic deformation. As is well known, the total thermopower (TEP), which is called also Seebeck coefficient (S), of bulk bismuth samples at liquid helium temperature is the sum of diffusion $\left(\mathrm{S}_{d}\right)$ and phonon drag term $\left(\mathrm{S}_{g}\right)$ :

$$
S=S_{\mathrm{d}}+S_{\mathrm{g}}
$$

Systematic measurements on a set of bulk Bi samples with different sizes have shown the changes in magnitude of phonon drag thermopower with moderately varying the size of samples [8-11]. As concerns low-dimensional Bi samples, such as films, whiskers, and wires, there is a larger spread in the measurements of thermopower. Farther, a strong discrepancy was observed between experimental results for $\mathrm{Bi}$ wires with the same diameters obtained by different technological methods [12-16], see Table 1. In different cases of positive $S$, indicating p-type transport for Bi NWs composites [13] and for individual polycrystalline NWs [2] or negative $S$ of $\mathrm{Bi} / \mathrm{Al}_{2} \mathrm{O}_{3}$ arrays [14], the authors suggest that an uncontrolled impurities could easily influence the value and sign of $S$.

Both the low-temperature TEP of Bi whiskers [15], which is of the order of a few $\mu \mathrm{V} \mathrm{K}^{-1}$, and the giant Seebeck effect observed in Bi nanocomposites [16], have a strange feature: the sign of $S$ varies from sample to sample. These facts would imply that, in addition to classical or quantum size effects, other factors, such as structural defects and impurity must also be considered to understand anomalies found in the transport properties of small-diameter Bi wires.

The data presented for bulk Bi crystals oriented along the bisectrix axis indicated an intrinsically negative phonon drag contribution that dominates TEP at low temperatures $[9,10]$. Our previous investigations of individual glass-coated Bi wires oriented along the bisectix axis have revealed a large positive thermopower dominated by the 
diffusion effect [17]. The diffusive origin of the TEP was attributed to structural imperfections, which strongly diminish the phonon-electron contribution to the total TEP. The suppression of the electron contribution to TEP was explained by a mechanism based on the model of selective carrier scattering by potential barriers, advanced by Ravich [18]. These structural imperfections with charge-selective scattering properties can be used to improve the efficiency of thermoelectric nanowires by removing some group of competing charge carriers from transport. Unfortunately, in most cases, structural defects can be naturally generated during the specific process of nanowire preparation. Some significant changes in the quality and parameters of Bi NWs can be obtained using different external impacts, such as thermal annealing, electric field effect, deformation, and magnetic field.

The final goal of the the present work is to understand the effect of size, thermal treatment, and uniaxial strain on the transport properties in order to improve the quality of Bi NWs. We report the results of the uniaxial strain effect on the thermopower and conductibility in glass-coated individual Bi wires. By combining thermal annealing, uniaxial deformation, and magnetic field, we have succeeded in conducting with thermoelectric parameters and in controlling with band structure by means of thermomagnetic measurements.

\section{Experimental Material and Method}

Long Pyrex-coated Bi wires were fabricated using the same improved variant of the Taylor method. This method, which is presently known as the glass-coated melt spinning method [19] consists in melting a metal in a glass tube by the induction heating and drawing a glass capillary in which the molten metal is entrapped. This method makes it possible to easily obtain long elastic Pyrex-coated wires protected from oxidation (Fig. 1). The wire axis makes an angle of about $19^{\circ}$ with the bisector axis $C_{1}$ in the bisector-trigonal plane $C_{1} C_{3}$. This orientation is the same as that observed in $\mathrm{Bi}$ nanowire arrays by Lin et al. [1]. The given crystallographic orientation along bisector axis is the preferential growth orientation that dominates in most prepared thin $\mathrm{Bi}$ wires and nanowire arrays [3,12-14]. Due to the high elasticity of Pyrex capillaries,

Fig. 1 SEM image of cross section of $\mathrm{Bi}$ wire with diameter of $2.0 \mu \mathrm{m}$

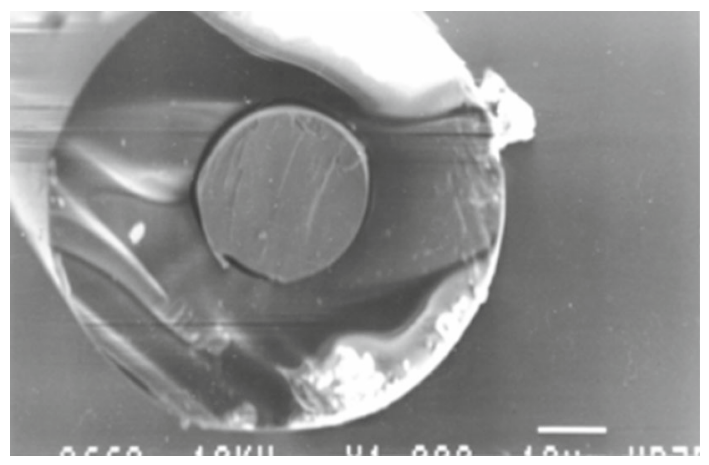


Fig. 2 Schematic image of the bottom part of the stretching rod (Color figure online)

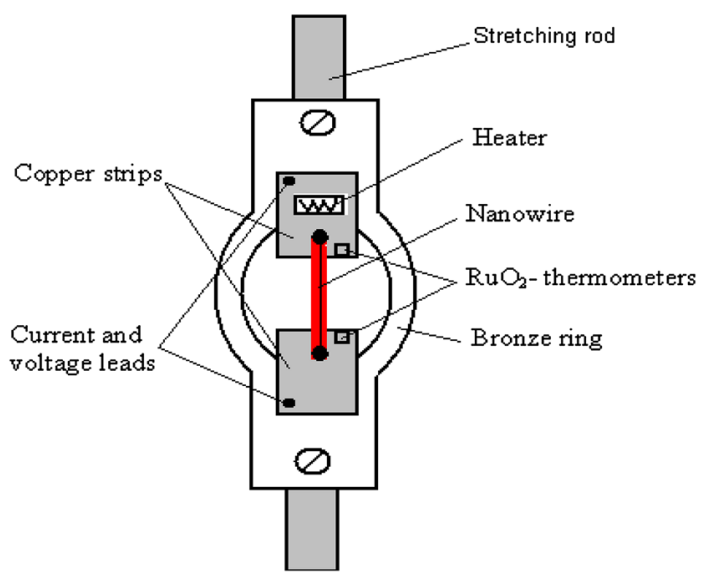

the limit of elastic deformation of the glass-coated Bi NWs attains $\varepsilon=3.5 \%$ (for comparison: for Bi whiskers [15] $\varepsilon=2.0 \%$ and for bulk Bi samples [20] $\varepsilon=0.4 \%$ ).

Due to small dimensions of the glass-coated NWs, it was not possible to apply mechanical loading directly to them. For the measurements under a uniaxial strain, the glass-coated NWs with the length $L_{0}=2.0-3.0 \mathrm{~mm}$ were mounted on an elastic bronze ring in a special insert with a stretching device similar to the method used for whiskers [15]. The difference is that instead of the $\mathrm{Cu}-\mathrm{Fe}$ thermocouples [15] were used $\mathrm{RuO}_{2}$ thermometers for more precise measurement in magnetic field. The wire was mounted on two separated copper strips (electrical contacts to the wire ends were soldered with Wood's alloy, covered and consolidated with epoxy). Copper strips are fixed by thin epoxy layer on an elastic bronze ring in a special insert with a stretching device. The bottom edge of the ring was rigidly fixed, the upper edge was connected with the stretching rod. The ring was stretched with a screw-nut mechanism, calibrated at room temperature and adjusted at low temperature. Schematic image of the bottom part of the stretching rod is presented in Fig. 2. Stretching was directed along the wire axis, i.e. close to the bisector axis $C_{1}$. The measurements of resistance of NWs with diameters of $d=0.1-3.0 \mu \mathrm{m}$ were carried out using a two-probe method. Resistance variation was defined as $\Delta R / R=\left(R-R_{0}\right) / R_{0}$, where $R_{0}$ is the value of resistance in non-deformed state. Strain variation was defined as $\varepsilon=\left(L-L_{0}\right) / L_{0}$, where $L_{0}$ is the length of the wire in a non-deformed state. Low $d c$ currents $(0.1 \leq I \leq 1 \mu \mathrm{A})$ were used to make sure that the voltage of the sample was a linear function of the applied current. The thermopower was measured by a steady-state technique using a miniature heater and two $\mathrm{RuO}_{2}$ thermometers. To have a reasonable signal-to-noise ratio, we were compelled to apply a relatively large temperature gradient, $0.4 \mathrm{~K} \leq \Delta T \leq 0.8$ $\mathrm{K}$. The magnetic-field dependences of the TEP $(B \| \Delta T)$ and electric resistance $(B \| I)$ were taken in separate runs.

The temperature dependences of the thermopower and resistance were measured in a closed-cycle refrigerator operating in a temperature range of 4-300 K. The thermopower measurements were carried out in an atmosphere of exchange gas. The measurements of magnetoresistance (MR) and magnetothermopower (MTEP) were 
performed in an appropriate cryostat in a magnetic field up to $14 \mathrm{~T}$ in a Bitter magnet at the International Laboratory of High Magnetic Fields and Low Temperatures, Wroclaw, Poland.

\section{Results and Discussion}

\subsection{Galvanomagnetic Size Effect}

The measurements of magnetoresistance and magnetothermopower were carried out for the set of wires with diameters of $0.1-3.0 \mu \mathrm{m}$ in a magnetic field parallel to the axis of the wire. The alignment of wire axis with magnetic field was confirmed by comparing positions of the peaks of Shubnikov-de Haas $(\mathrm{SdH})$ oscillations on the longitudinal magnetoresistance for $\mathrm{Bi}$ wire with theirs positions on the longitudinal magnetoresistance for bulk Bi crystals in the same crystallographic direction. Misalignment in the parallel field may be to within $1 \%$. Reproducibility of measurements was confirmed by comparing curves of longitudinal magnetoresistance in the nondeformed state before to load and after removal of stretching load. Within the limits of experimental error, the frequencies and phases of the observed $\mathrm{SdH}$ oscillations are in good agreement with the accepted values for bulk bismuth crystals oriented along bisector axis: $F_{1 e}=1.26 \mathrm{~T} ; F_{2 e, 3 e}=2.73 \mathrm{~T}$ and $F_{\mathrm{h}}=14.9 \mathrm{~T}$ (inset in Fig. 3).

In Fig. 3 are presented the longitudinal MR and longitudinal MTEP data for 180 $\mathrm{nm} \mathrm{NW}$ at temperatures of 4.2 and $5.0 \mathrm{~K}$, respectively. Longitudinal MR exhibits non-

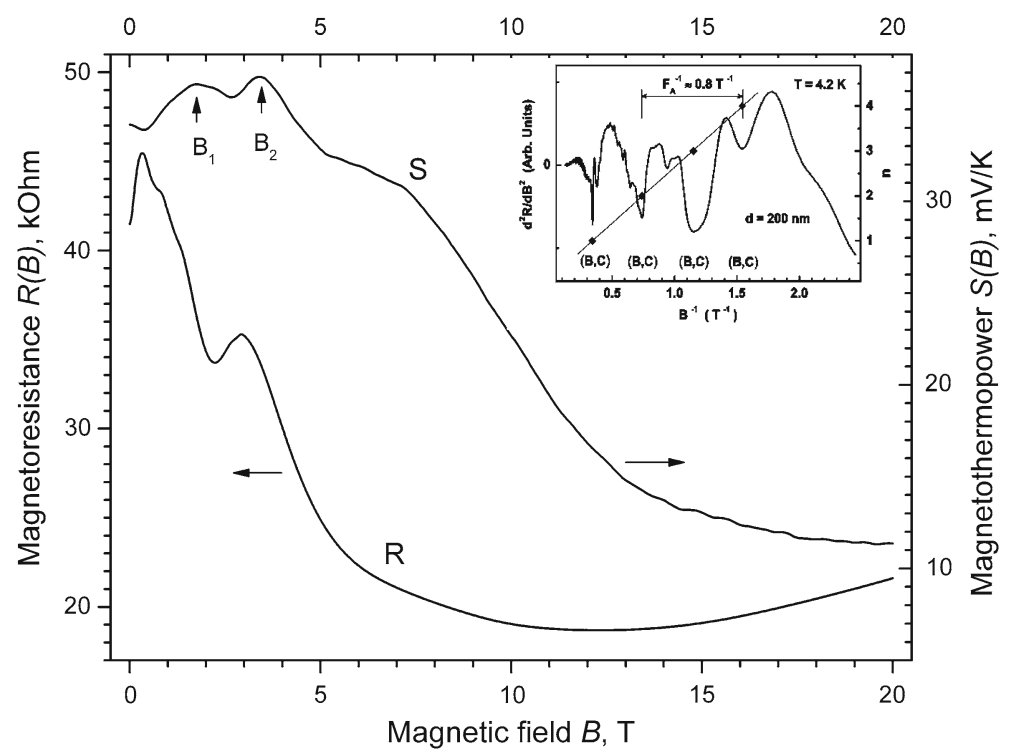

Fig. 3 The magnetoresistance and magnetothermopower for Bi nanowire with $d=180 \mathrm{~nm}$ at $T=4.2$ and $5.0 \mathrm{~K}$, respectively. The vertical arrow $B_{2}$ indicates the magnetic field at which the cyclotron hole orbit equals the wire diameter. Inset second derivative $\partial^{2} R / \partial B^{2}$ as a function of $1 / B$ for the MR curve. The straight line (right $y$-axis) is a fit to the plot of the values of the peak number $n$ versus the values of $1 / B$ at the respective minima of $\partial^{2} R / \partial B^{2}$ for the heavy electrons and indicates frequency of $2.7 \mathrm{~T}$ 
monotonic field dependence consistent with previous reports on bismuth wires [21]. Longitudinal magnetoresistance achieves its maximum value in a magnetic field $B_{\text {max }}$, where the cyclotron radius of electrons $r_{c}$ equals the wire radius, which is consistent with the theory of Chambers [22]. The cyclotron radius is given by: $r_{c}=h k_{F} / 2 e B_{\max }$ where $h$ is Planck constant, $k_{F}$ is the Fermi wave vector for the appropriate carrier, heavy or light electrons or holes, and $e$ is the electron charge. As the field increase above $B_{\max }$ the MR starts decreasing because the radius of the carrier trajectory is smaller than the wire radius, causing a decrease in the boundary scattering. The rapid decrease in MR masks the oscillatory component, but several peaks from Shubnikovde Haas $(\mathrm{SdH})$ oscillations can be seen in a magnetic field of $B>B_{\max }$.

Inset in Fig. 3 illustrates an intermediary step in the analysis of the frequencies of the $\mathrm{SdH}$ oscillations on magnetoresistance curve. The frequency of oscillations, $F$, has been obtained from the slope of the peak number $n$ against the reciprocal field $(1 / B)$ at which the $n-$ th peak occurs. The straight line gives for the heavy electrons from two equivalent pockets $\mathrm{B}$ and $\mathrm{C}$ a frequency of $F_{B C}=2.7 \pm 0.1 \mathrm{~T}$ Since the spin-splitting factor for these electrons is close to unity, their field of quantum limit practically coincides with their frequency, that is $F_{B C}=B_{q . l i m i t} \approx 2.7 \mathrm{~T}$. The trace of the presence of light A-electrons with a frequency of $F_{A} \approx 1.3 \mathrm{~T}$ is related to some modulation of the amplitude of heavy-electron oscillations $\left(F_{B C} \sim 2 F\right.$ A $)$ visible in the figure from inset. Thus, at field $\mathrm{B}>3 \mathrm{~T}$ the quantum limit for all the electrons in magnetic field parallel to bisector axis has already been reached, which corresponds to the statements from reference [13].

The thermopower (curve $\mathrm{S}$ in Fig. 3) is positive in all magnetic fields. With increasing magnetic field MTEP increases and passes through two maxima at $B_{1} \approx 1.7 \mathrm{~T}$ and $B_{2} \approx 3.6 \mathrm{~T}$. Unlike the MR curve that exhibited some weak $(\mathrm{SdH})$ oscillations from electrons, there are no oscillations from electrons on MTEP. At the high magnetic field where only the holes are still in the quasi-classical range of magnetic fields, and one can observe weak oscillations of $S$ resulting from the quantization of their spectrum.

As we shown [17], the increase in the positive value of TEP takes place in the magnetic field region where condition: $\left(l_{h} d\right)^{1 / 2}>r>d / 2$ is satisfied for holes $\left(l_{h}\right.$ is mean free path of holes, $d$ is diameter of wire) and one of the more-pronounced peak at $\mathrm{B}_{2} \approx 3.6 \mathrm{~T}$ on magnetothermopower curve is the artifact of size effect induced by the diffusion reflection of holes on the wire wells: $B_{2}=2 c p_{F} / e d$, where $p_{F}$ is the Fermi momentum of hole.

Under close examination of MTEP versus magnetic field and taking into account of all the peaks on the magnetothermopower curve, FFT analysis yield a frequency of $F=3.7 \mathrm{~T}$, which is absent in the MR data.

At magnetic field range of 12-15 T we observed the presence of some weak oscillations in the thermopower. According to the analysis of magnetoresistance curves, the quantum limit for all the electrons has already been reached at field $\mathrm{B} \approx 2.7 \mathrm{~T}$, therefore we can unambiguously claim that the peaks and wiggles at $\mathrm{B}>3 \mathrm{~T}$ may not be related to quantum oscillations from electrons. Currently we should recognize that we can not explain the nature of the wiggles in the thermopower at magnetic field around $15 \mathrm{~T}$.

The performed measurements of MTEP on the set of the NWs with diameters of $130-350 \mathrm{~nm}$ reveals the shift of the peak $B_{2}$, due to size effect of holes reflection, to 


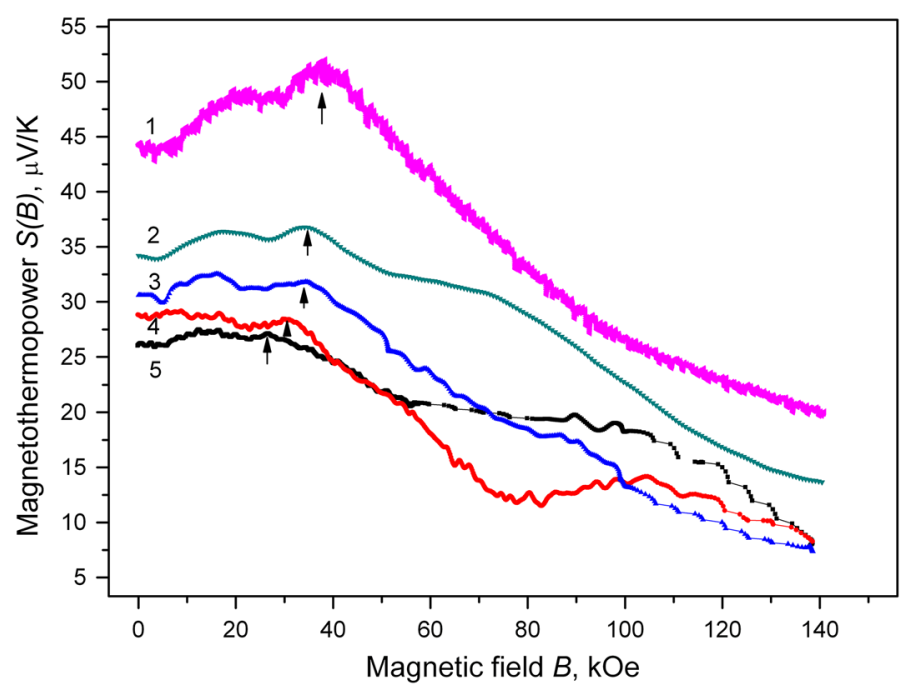

Fig. 4 Magnetothermopower for Bi NWs of various diameters: 1-130, 2-180, 3-220, 4-300, 5-350 nm at $T=5 \mathrm{~K}$. The vertical arrows indicate position of $B_{2}$ as the artifact of size effect induced by the diffusion reflection of holes on the wire wells. The position of $B_{2}$ decreases from $B_{2}=3.8 \mathrm{~T}$ for the 130-nm wire down to $B_{2}=2.7 \mathrm{~T}$ for the wire with $d=350 \mathrm{~nm}$ (Color figure online)

lower magnetic fields with increasing wire diameter (Fig. 4). Decrease of the magnetic field value $B_{2}=3.8 \mathrm{~T}$ for the $130-\mathrm{nm}$ wire down to $B_{2}=2.7 \mathrm{~T}$ for the wire with $d=$ $350 \mathrm{~nm}$ is consistent with the values calculated by the formula $B_{2}=2 c p_{\mathrm{F}} / e d$.

The absence of well-defined size-effect features corresponding to electrons on $S(B)$ indicates that the reflections of the majority of these carriers at the wire boundary are specular [23].

\subsection{Diffusion Transport Mechanism}

Temperature dependences of the thermopower for NWs with $d=100-350 \mathrm{~nm}$ exhibit a quasi-linear behaviour with a different positive slope in temperature ranges of 5$20 \mathrm{~K}$ (curves 1-3 and inset a) in Fig. 5). Considering the quasi-linear dependence of $S(T)$, the observed TEP was associated with the diffusion transport mechanism. According to [17], the diffusive origin of the TEP was attributed to the limited diameter and to structural imperfections within the nanowire which strongly diminish the contribution from electrons or from the phonon-electron interaction to the total thermopower. The suppression of the electron contribution to TEP was explained by a mechanism based on the model of selective carrier scattering by potential barriers, where the electrons are scattered much stronger than holes, which eventually influences the mobility of electrons: $\mu_{\mathrm{e}}<\mu_{\mathrm{h}}$ [18]. The predominance of the diffusion positive thermopower $S_{\mathrm{d}}$ in the total one was observed for the set of thin NWs with diameters $d=100-350 \mathrm{~nm}$ characterized by low residual resistance ratio $R R R=0.5-$ 2.0. On the other hand, we observed the predominance of the phonon drag term for the thicker wires with $d>600 \mathrm{~nm}$ and $R R R=25-30$ (Fig. 5, curve 4 and curves 5-7 in inset). 


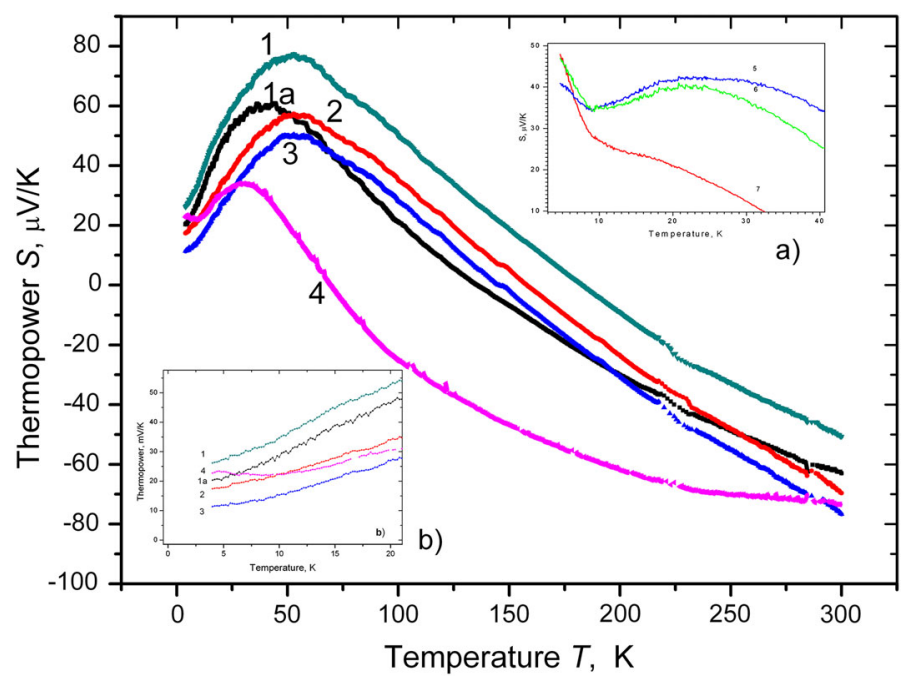

Fig. 5 Temperature dependences of the thermopower for as-prepared and thermal annealed Bi NWs of various diameters: 1-120 nm (as-prepared), 1a-120 nm (after thermal treatment), 2-250, 3-350, 4-650 nm. Inset a 5-0.8, 6-1.1, 7-3.0 $\mu \mathrm{m}$. Inset $\mathbf{b}$ Expanded version of $\mathrm{S}(\mathrm{T})$ dependences in the temperature range 4.2-20 K (Color figure online)

This can be attributed to an increase in the ratio of electron to hole mobility, which in turn can be accounted for by either a diminished influence of the surface scattering of electrons (due to an increase in the wire diameter) or a decrease in the concentration of structural imperfections (due to technological conditions in the preparation of thicker wires).

The temperature dependences of TEP shown in Fig. 5 for as-prepared and thermal annealed NW reveal an increase in the negative contribution to the thermopower after thermal treatment. The observed increase of $R R R$ on the $R(T)$ for the same wire after thermal treatment (Fig. 6) testifies to an improved quality of annealed NWs. This supports the idea that a low $R R R$ and a negative temperature coefficient of resistance $(T C R)$ are not caused only by the classical or quantum size effect: a large value of resistivity at $T=4.2 \mathrm{~K}$ may be rather an evidence for some defects inside the asprepared wires. Taking into consideration the increase of negative contribution to the thermopower (Fig. 5) with increasing residual resistance ratio (Fig. 6) for nanowires with the same diameter but improved quality after thermal treatment, we can conclude that, in this case, the sensitivity of TEP to the structural imperfections within nanowire is more pronounced than to the surface scattering on walls. This is the case of indirect connection between the diameter and structure of the wire and this dependence is defined by the technology.

It should be mentioned that the disadvantages of used melt spinning method are the necessity to overheat the metal in order to soften the glass tube, and also the high rate of cooling in the process of solidification of the molten $\mathrm{Bi}$ core, which can produce some structural defects. The fact of the generation of defects, and its dependence on the diameter was reported in the theoretical model [24], which describes the generation of dislocations in the composites of wire form. The model specified that occurrence 


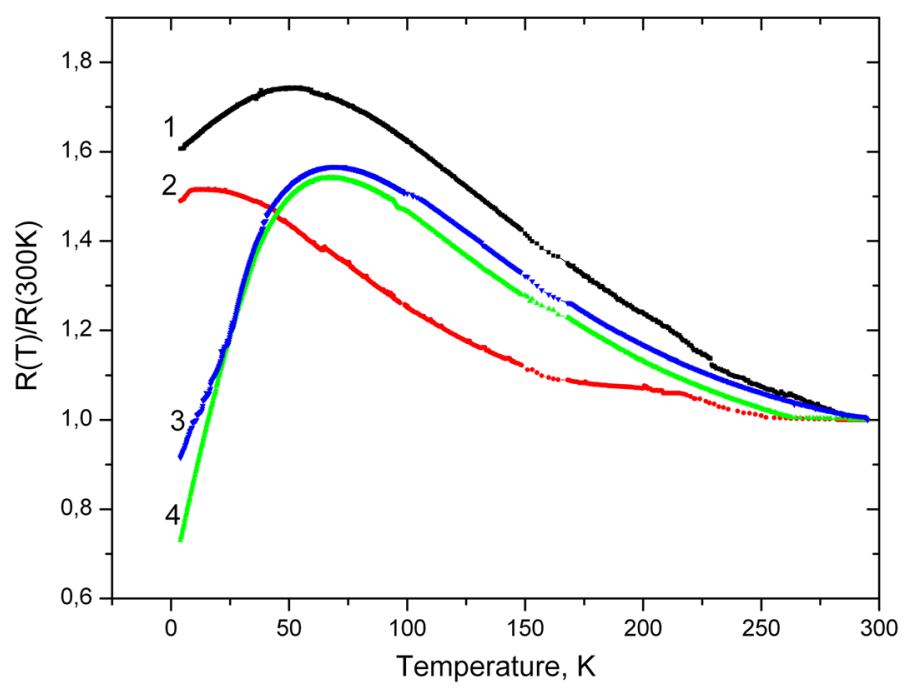

Fig. 6 Temperature dependence of resistance normalized to the resistance at $300 \mathrm{~K}$ of different diameters for as-prepared Bi NWs $1 d=90 \mathrm{~nm}, 3 d=120 \mathrm{~nm}$ and thermal annealed $2 d=90 \mathrm{~nm}, 4 d=120 \mathrm{~nm}$ (Color figure online)

of the defects is energetically favorable in the wire composites when their geometric parameters are in certain ranges, namely there is a critical thickness above or below which dislocations may be formed. If in the process of solidification, the softened glass-coated $\mathrm{Bi}$ wire is regarded as a composite, there is a critical diameter of $\mathrm{Bi}$ core below which structural defects may be generated due to the cylindrical geometry of wire composite as was suggested [24]. Is natural to suppose that generated defects have an impact on the carrier scattering at low temperatures.

In our early published paper [17] in order to explain the positive sign of the diffusion TEP at low temperature, which in pure Bi may arise only from the difference in electron and hole mobility, were analyzed the various mechanisms that could scatter electrons more effectively than holes.

In the preceding analysis we took into account that in compensated semimetals, any type of surface or bulk defects that scatter electrons more effectively than holes is likely to enhance the positive contribution to the total $S$. Since the TEP remains hole-like in strong magnetic fields, where all the electrons moves quasi-one-dimensionally parallel to the wire axis, the surface scattering is probably not a mechanism determinative of the $S$ sign. The suppression of the electron contribution seems to be related to the complexity of the wire microstructure. A possible mechanism responsible for the enhanced values of the positive TEP may be based on the model of selective carrier scattering by potential barriers [18]. Such barriers usually arise at grain boundaries, where some changes in the potential occur due to the defects, local stresses, etc. In this context, our Bi nanowires can be regarded as consisting of a number of elongated crystallites separated by 'transverse' boundaries, which act as energy barriers with charge-selective properties: the electrons are scattered much stronger than holes. 
It was interesting to note that to explain a very strong specular scattering of electrons at the Bi surface Tsoi and co-workers [23] used the concept of 'electron-reflecting hole-transmitting' potential barriers originating from surface states and band bending effects. Surface states (negatively charged) are considered to be responsible for the band bending, which creates a barrier reflecting electrons and, at the same time, provides conditions favorable for holes to be scattered by the surface roughness. In order to interpret our experiments, one has to assume that the potential barriers with the similar charge-selective scattering properties are present not only at the Bi/Pyrex interface, where they suppress almost completely the diffuse surface scattering of electron carriers, but also near the crystallite boundaries within the nanowire, where they cause backscattering of electrons, producing a large contribution to the residual resistivity. Note that the presence of internal electron-reflecting hole-transmitting barriers may lead to a localization-like behavior for the electrons and a metallic behavior for the holes. A similar scenario in the sense of localized electrons and delocalized holes has been invoked by Azbel [25] to interpret the metallic conductivity observed in the experiments on high-resistivity Bi and Bi-6 at\% Sb whiskers with $d=140-210 \mathrm{~nm}$. The above information allows us to conclude that the $\mathrm{SdH}$ oscillations in $\mathrm{Bi}$ nanowires originate from the crystallites with bulk-like carrier densities, while the large values of the positive thermopower and resistivity are determined by the boundaries between crystallites, which reduce the mobility of electrons to such an extent that the majority of the conductivity comes from holes. Perhaps this model is not perfect, but we can not yet explain the dominance of the holes in the diffusive thermopower of compensated semimetal as $\mathrm{Bi}$ at low temperatures.

Then, in the framework of the accepted scenario of the localized electrons, one may suppose that potential barriers can be neglected if electrons get a necessary energy to liberate. A partial destruction of the barriers has been achieved by thermal annealing, which is shown in a decrease in the residual resistance (Fig. 6) and an increase in the negative contribution to TEP (Fig. 5).

In general, the emergence of the maxim on TEP is due to limited size of the wire when compared with the temperature dependence of TEP for balk Bi along the bisectrix axis, where maxim is absent [13]. As we clamed on diffusive mechanism of TEP in the temperature range of 4.2-25 K, the emergence of maxim on TEP may be due to the competition between electrons and holes contribution to the diffusive TEP. Mobility of holes rises with temperature, whereas electrons are still "localized" by potential barriers. After maxim of TEP electrons get a necessary energy and their mobility overcome holes mobility. In particular, we can speculate that the peak of TEP around $50 \mathrm{~K}$ partially reflects a transition from the localization-like region for electrons to the region of the domination of "liberated" electrons. The other part of contribution to the TEP peak is due to surface scattering of electrons, the specularity of which decreases approaching the liquid nitrogen temperature. The thermal energy needed to liberate a "localized" electron, shifts to lower temperatures for wires with improved quality after thermal treatment (curve 1a, Fig. 5). The temperature dependence of TEP in a range of 50-300 $\mathrm{K}$ becomes similar to the dependence of TEP for bulk Bi along the bisectrix axis [13]; therefore, the drop of TEP above $50 \mathrm{~K}$ is due to a decrease in the hole contribution in favor of the electron contribution to the total diffusive TEP. 


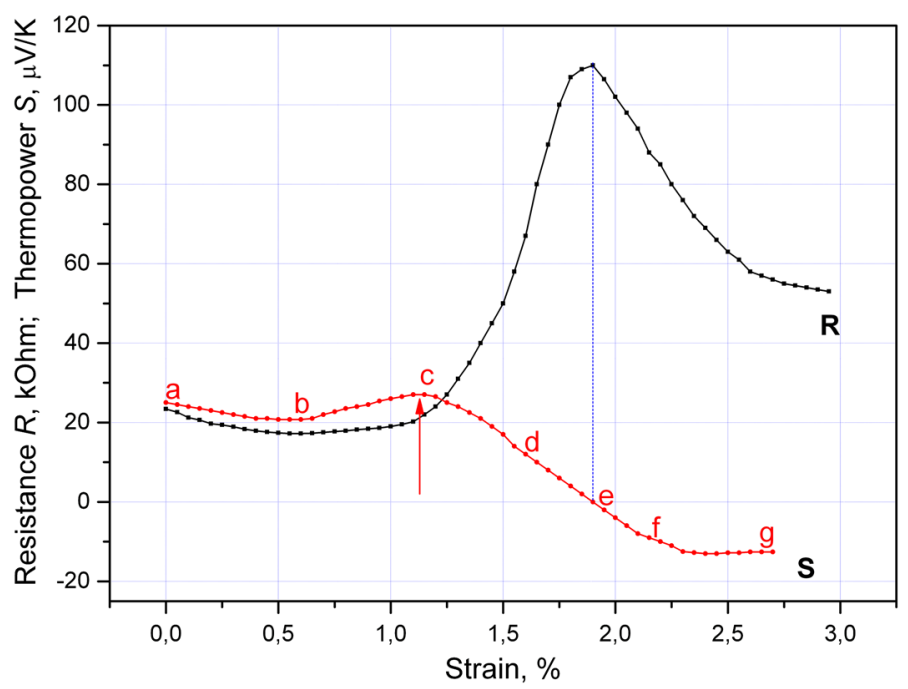

Fig. 7 Resistance $R(\varepsilon)$ (curve $R$ ) and thermopower $S(\varepsilon)$ (curve $S$ ) dependences as a function of applied strain for $300 \mathrm{~nm} \mathrm{Bi}$ wire at $4.2 \mathrm{~K}$. The vertical arrow (c-point) shows the peak due to ETT on the straindependence of TEP. The vertical dashed line connects the zero-point of TEP (e-point) and the maxima point of resistance (Color figure online)

\subsection{Bi Wires Under Strain}

Note that an increase in the contribution of electrons to TEP up to a sign change from positive to negative was observed in our earlier investigations on $\mathrm{Bi}$ wires under the uniaxial strain [26,27]. Measurements under strain were made in the limit of elasticity. The reproducibility of the measurements was confirmed by comparing curves of longitudinal magnetoresistance and magnetothermopower in the non-deformed state before to load and after removal of stretching load. In a rare cases (not more than $5 \%$ ), some of the samples under strain passed in the plasticity state, which was accompanied by a sharp change in the resistance and thermopower. Note that detailed results of investigations of $\mathrm{Bi}$ wires in the plasticity state may be the subject of another paper.

In the continuation of the investigations of the strained $\mathrm{Bi}$ NWs were measured the dependences of resistance and thermopower on strain $(0 \leq \varepsilon \leq 3 \%)$ and temperature ( $4.2 \mathrm{~K} \leq T \leq 300 \mathrm{~K})$. In general, the shapes of the resistance dependences on strain $R$ $(\varepsilon)$ resemble each other for the wires of all the investigated diameters, a characteristic behavior at $T=4.2 \mathrm{~K}$ is presented in Fig. 7, curve R.

The strain dependences of the TEP are quiet different for thinner and thicker wires in the investigated range of diameters (Fig. 8). Two types of behavior for two sets of samples were revealed: first type for NWs with diameters $0.1 \leq d \leq$ and second type for wires with $0.35<d<3.0 \mu \mathrm{m}$. The application of strain upon the wires of the second type $(d>0.35 \mu \mathrm{m})$ does not produce any variation of the $S(\varepsilon)$ dependence up to a strong deformation (Fig. 8, curve 1,2). Only above $\varepsilon=2.0 \%$, the $S(\varepsilon)$ decreases and trends to zero. The second type of behavior is developed for wires when phonon drag contribution is dominant in the total TEP. In the case of first type wires $(d \leq 0.35 \mu \mathrm{m})$ 


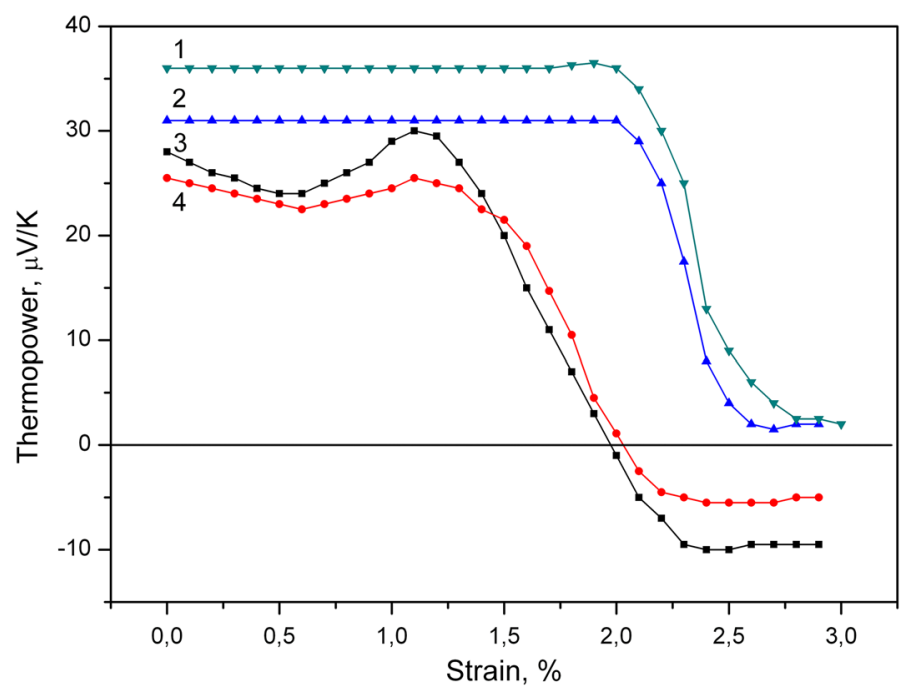

Fig. 8 Strain-dependence of TEP for Bi wires of different diameters at 4.2 K: 1-2.4, 2-1.0, 3-0.18, 4-0.35 $\mu \mathrm{m}$ (Color figure online)

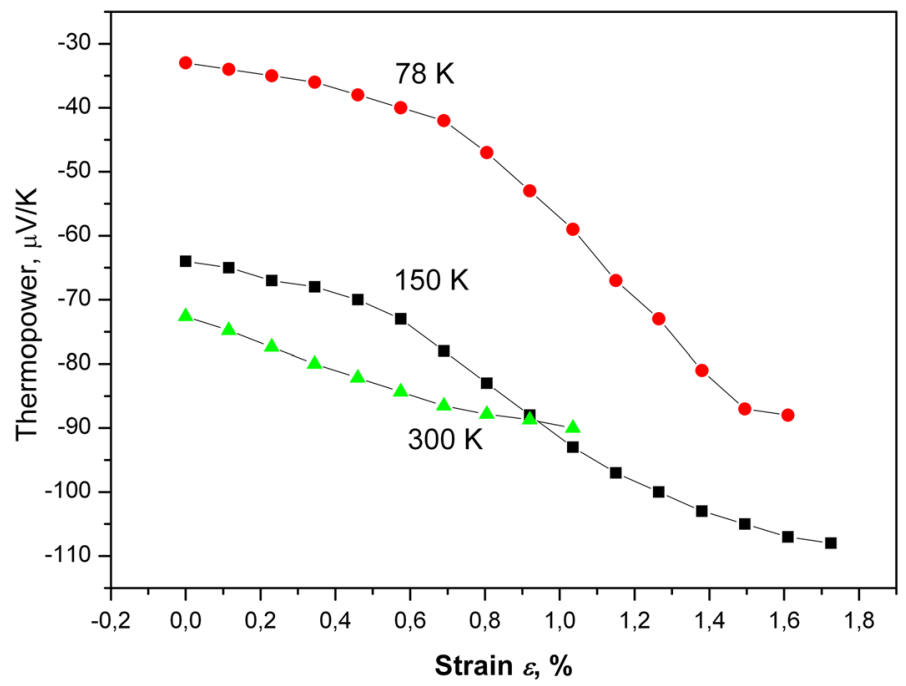

Fig. 9 Strain-dependences of the thermopower of Bi wire of diameter $d=1.0 \mu \mathrm{m}$ at different temperatures (Color figure online)

the $S(\varepsilon)$ dependences exhibit a non-monotonous behavior, which indicates a complex charge transport mechanism (Fig. 7, curve S and Fig. 8, curve 3, 4). The initial positive TEP smoothly decreases, goes through fine minima and increases up to a peak around strain of $1.1 \%$ with the following sharp decrease, a change of sign, and the nearly saturation behavior under a higher strain. Note that a non-monotonous behavior is inherent in the wires in which TEP is dominated by the diffusion transport mechanism. 


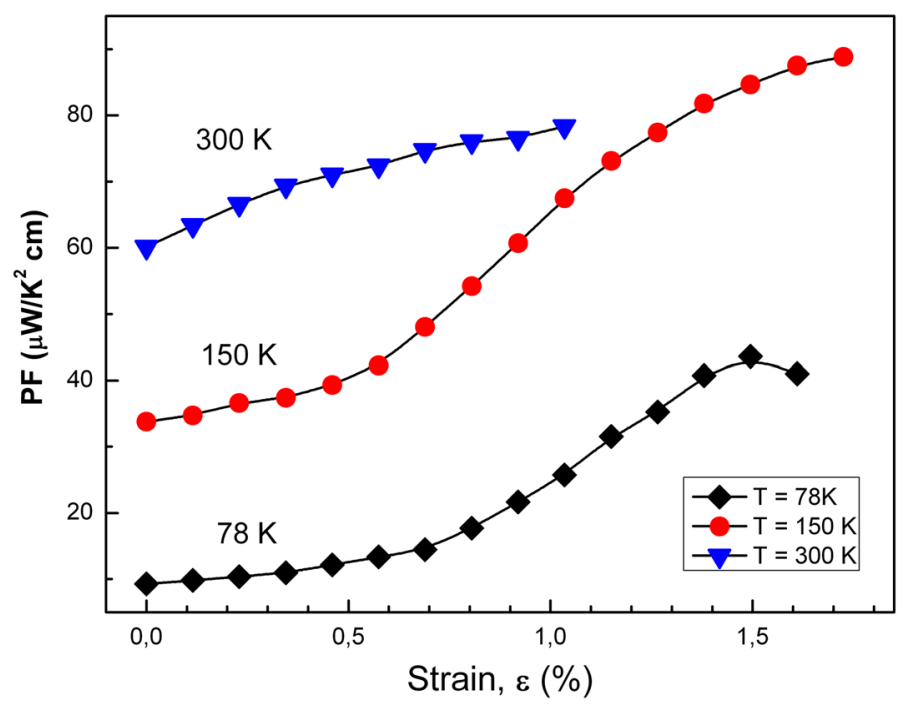

Fig. 10 Strain-dependences of the thermoelectric power factor (PF) of Bi wire $d=1.0 \mu \mathrm{m}$ at different temperatures (Color figure online)

This complex behavior is representative of a competition among the different scattering mechanisms for the carriers and balances between the diffusive transport of electron or hole parts of the Fermi surface that changed during straining.

In the region of high temperatures $(T \approx 100-300 \mathrm{~K}$, Fig. 5$)$, where the mechanism of electron transport is operating in the thermopower, the absolute value of the negative TEP increases under strain ( $T \approx 77-300 \mathrm{~K}$, Fig. 9 ) while resistance smoothly decreases. Some estimated values for the thermoelectric power factor were obtained at strain $\varepsilon=1.5 \%: S^{2} / \rho=57 \mu \mathrm{W} / \mathrm{cm} \mathrm{K}^{2}$ for $T=78 \mathrm{~K}$, and $89 \mu \mathrm{W} / \mathrm{cm} \mathrm{K}^{2}$ for $T \approx$ $150 \mathrm{~K}$ (Fig. 10). These values are fairly high for Bi nanowires with strong quantum confinement.

\section{Conclusions}

Investigated Bi NWs were prepared by the melt spinning method that makes it possible to easily obtain long elastic Pyrex-coated wires, protected from oxidation. One of the disadvantages of this method is the high rate of cooling in the process of solidification of the molten Bi core, which can produce some structural defects. Thus, specific imperfections inherent in thin NWs are defined by the technology. If a softened glass-coated $\mathrm{NW}$ is regarded in the solidification process as a composite, there is a critical diameter below which structural defects can be generated, due to the cylindrical geometry of the NWs composite, as was suggested [24]. For Bi wires, the critical diameter is around $350 \mathrm{~nm}$, below which structural defects cause the appearance of internal electronreflecting hole-transmitting barriers, which can lead to a localization-like behavior for the electrons and a metallic behavior for the holes. 
Some improvements of the NWs quality were achieved by means of thermal annealing that has an effect on the lowering of potential barriers and the liberation of electrons for transport.

Note that an increase in the contribution of electrons to TEP up to a sign change from positive to negative was observed at low temperature for Bi wires under the strain. A fairly high value of thermoelectric power factor $\left(S^{2} / \rho=89 \mu \mathrm{W} / \mathrm{cm} \mathrm{K}^{2}\right)$ was found in the temperature range of 80-300 K, where the dominant mechanism contributing to the thermopower is diffusive thermoelectric generation with electrons as the majority carrier.

The effect of strain in the NWs is more complicated at the liquid helium temperature where the hole transport is dominant. However, the hole-dominated transport can be transformed into the electron-dominated transport through smooth manipulations Fermi surface by applying strain. The observed trend to negative values of thermopower under strain is representative of a slightly increasing of the mobility of electrons and the saturation-like behavior of negative TEP under a high strain is the result of competition among the different scattering mechanisms for the heat carriers and balances between the electron and hole phonon drag contribution.

The presented results on the effect of uniaxial pressure on the thermoelectric properties of Bi wires can be useful if they are considered in applying the "pressure tune" method for the synthesis of thermoelectric materials.

Acknowledgments We would also like to thank Dr L. Konopko from IIEN for assistance in conducting some measurements.

Open Access This article is distributed under the terms of the Creative Commons Attribution License which permits any use, distribution, and reproduction in any medium, provided the original author(s) and the source are credited.

\section{References}

1. Y.-M. Lin, X. Sun, M.S. Dresselhaus, Phys. Rev. B 62, 4610 (2000)

2. A. Boukai, K. Xu, J.R. Heath, Adv. Mater. 18, 864 (2006)

3. M.J. Gurvitch, J. Low, Temp. Phys. 38, 777 (1980)

4. N.P. Armitage, R. Tediosi, F. Levy, E. Giannini, L. Forro, D. van der Marel, Phys. Rev. Lett. 104, 237401 (2010)

5. T.W. Cornelius, M.E. Toimil, Molares, R. Neumann, S. Karin, J. Appl. Phys. 100, 114307 (2006)

6. F. Chen, K.L. Stokes, R. Funahashi, Appl. Phys. Lett. 81, 2379 (2002)

7. S.V. Ovsyannikov, V.V. Shchennikov, G.V. Vorontsov, A.Y. Manakov, A.Y. Likhacheva, V.A. Kulbachinsckii, J. Appl. Phys. 104, 053713 (2008)

8. J.-P. Issi, J.H. Manges, Phys. Rev. B 6, 4429 (1972)

9. C. Uher, W.P. Pratt Jr, J. Phys. F 8, 1979 (1978)

10. J. Boxus, J.-P. Issi, J. Phys. C 10, L397 (1977)

11. J. Boxus, C. Uher, J. Heremans, J.-P. Issi, Phys. Rev. B 23, 449 (1981)

12. P.P. Bodiul, D.V. Gitsu, V.A. Dolma, M.F. Miglei, G.G. Zegrea, Phys. Status Solidi (a) 53, 87 (1979)

13. J. Heremans, C.M. Thrush, Phys. Rev. B 59, 12579 (1999)

14. M. Y-, O. Lin, S.B. Rabin, J.Y. Cronin, Ying, M.S. Dresselhaus, Appl. Phys. Lett. 81, 2403 (2002)

15. Yu.P. Gaidukov, Sov. Phys.-Usp. 27, 256 (1984); Usp. Fiz. Nauk 142, 571.

16. J.P. Heremans, C.M. Thrush, D.T. Morelli, M.C. Wu, Phys. Rev. Lett. 88, 216801 (2002)

17. A.D. Grozav, E. Condrea, J. Phys. 16, 6507 (2004)

18. Y.I. Ravich, in CRC Handbook of Thermoelectrics, vol. 7, ed. by D.M. Rowe (CRC Press, New York, 1995), pp. 67-73 
19. M. Hagiwara, A. Inoue, in Production Techniques of Alloy Wires by Rapid Solidification in Rapidly Solidified Alloys, ed. by H.H. Liebermann (Dekker, New York, 1993), p. 141

20. O.P. Hansen, I.F. Mikhail, MYu. Lavrenyuk, N. Ya, Minina J. Low Temp. Phys. 95, 481 (1994)

21. N.B. Brandt, D.V. Gitsu, V.A. Dolma and Ya.G. Ponomarev, Sov. Phys. JETP 65, 515 (1987); Zh. Eksp. Teor. Fiz. 92, 913.

22. R.G. Chambers, Proc. R. Soc. Lond. Ser. A 202, 378 (1950)

23. V.S. Tsoi and I.I. Razgonov, JETP Letters 23, 92 (1976); Pis. Zh. Eksp. Teor. Fiz. $23,107$.

24. M. Ya, I.A. Ovidko, A.G. Sheinerman, J. Phys. 12, 5391 (2000)

25. A.M. Ya, Phys. Rev. B 27, 3852 (1983)

26. E. Condrea, A.D. Grozav and N.I. Leporda, Proceedings of the 6th European Workshop on Thermoelectrics of the European Thermoelectric Society 2001 (Freiburg, Germany, Sept. 2001) (Freiburg: Fraunhofer Institute of Physical Measurement Techniques IPM).

27. P.P. Bodiul, V.F. Garabazhiu, E.P. Condrea, A.A. Nikolaeva, Czech. J. Phys. 46, 2417 (1996) 